\title{
In-Situ Frequency Trimming of SAW Resonator Using Conventional Crystal Resonator Fine Tuning Method with Gold Thin film Addition
}

\author{
S.M. Wang, Y.J. Wang, Chung-Lun Lo, M.C. Chao, C.W. Liu, C.W. Lin, C.L. Wang, and C.S. Lam \\ TXC Corporation, Ping Cheng City, Taoyuan County, Taiwan
}

\begin{abstract}
SAW devices are routinely frequency trimmed at the wafer level prior to the dicing process so to improve yield at the assembly level. The trimming can be achieved by using wet etching, reactive ion etching, ion beam milling, and other methods. It is also possible to trim the frequency of patterned SAW wafer by depositing dielectric thin films on the wafer but this method is usually done for the purpose of finger shorting protection from metallic particles. Reactive ion etching can be used to trim assembled SAW devices prior to encapsulation. This is seldom employed since it is considered costly, inefficient, and difficult to perform in-situ. In this paper we propose trimming assembled SAW resonator (SAWR) by using the conventional crystal resonator fine-tuning process with gold thin film addition. Nowadays, the fine-tuning process is a necessary step in crystal resonator production. We show that this is a simple and well-controlled method to trim assembled SAW resonator also. This method provides a wide trimming range and the gold film is thin enough not to short out the SAW transducers so that the SAWR's integrity can be maintained.
\end{abstract}

\section{Introduction}

Nowadays for high volume production of SAW devices, patterned wafers are routinely frequency trimmed to increase chip yield. The trimming can be achieved by using wet etching, reactive ion etching, ion beam milling, and other methods. Wet etching trims frequency upward since metal fingers become thinner. Reactive ion etching and ion beam milling trim frequency downward since substrate material is removed. One commercial frequency trimming system (S\&A 5911) uses the ion beam milling method to etch a complete wafer at one time (Fig. 1). Uniformity measurements (probing) are first made prior to milling. The system uses the data to etch different areas of the wafer different amounts of time and claims to improve the overall uniformity by a factor of 5. Typical etching time per quartz wafer is about 20 minutes $\left(\sim 100 \AA \mathrm{SiO}_{2}\right.$ removed). It is also possible to trim the frequency of patterned SAW wafer by depositing dielectric thin films on the wafer ${ }^{[1]}$ but this method is usually done nowadays for the purpose of finger shorting protection from metallic particles. Dielectric thin film deposition is also being used to achieve better temperature coefficient of delay (TCD) ${ }^{[2,3]}$. Reactive ion etch can be used to trim assembled SAW devices prior to encapsulation. This is seldom employed since it is considered costly, inefficient, and difficult to perform in-situ.

Recently a commercial system (S\&A 5910- similar to Fig. 1) became available to trim frequency of assembled SAW devices efficiently using ion beam milling. The system was modified from the conventional crystal resonator frequency trimming system except a high frequency network analyzer was employed since SAW devices usually operated at higher frequencies. The system etches 16 devices in about one minute $\left(\sim 100 \AA \quad \mathrm{SiO}_{2}\right.$ removed). This is the only commercially available in-situ SAW frequency trimming system the authors are aware of.

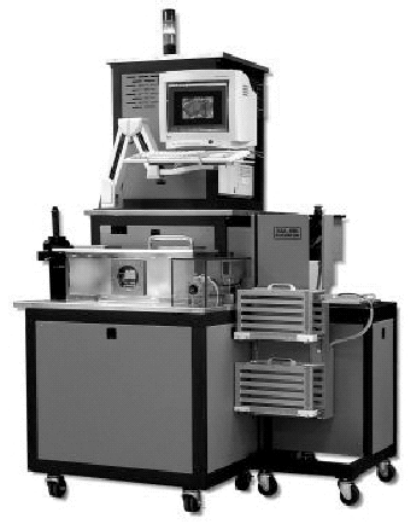

Figure 1. SAW Wafer Frequency Trimming S\&A 5911 System \& SAW Device Frequency Trimming S\&A 5910 System (Courtesy: Saunders \& Associates, Inc.)

In this paper we propose trimming assembled SAW resonator (SAWR) by using the conventional crystal resonator fine-tuning process with gold thin film addition. Nowadays, the fine-tuning process is a necessary step in crystal resonator production. We show that this is a simple and well-controlled method to trim assembled SAWR also. This method provides a wide trimming range and the gold film is thin enough not to short out the SAW transducers so that the SAWR's integrity can be maintained.

\section{Gold Thin Film on SAW Devices}

Effects of different thin films (metallic, dielectric, piezoelectric, etc.) on SAW devices were comprehensively summarized by Hickernell $^{[4]}$. Gold thin film effect on quartz substrate was studied by Cambon et al. earlier ${ }^{[5,6]}$ and the decrease of Rayleigh wave velocity and increase of attenuation loss verse increase of thin film thickness to the acoustic wavelength ratio was as shown in Fig. 2. According to Cambon et al., there were three distinct regions of influence related to the thin film growth, electrical conductivity, and mass of the films.

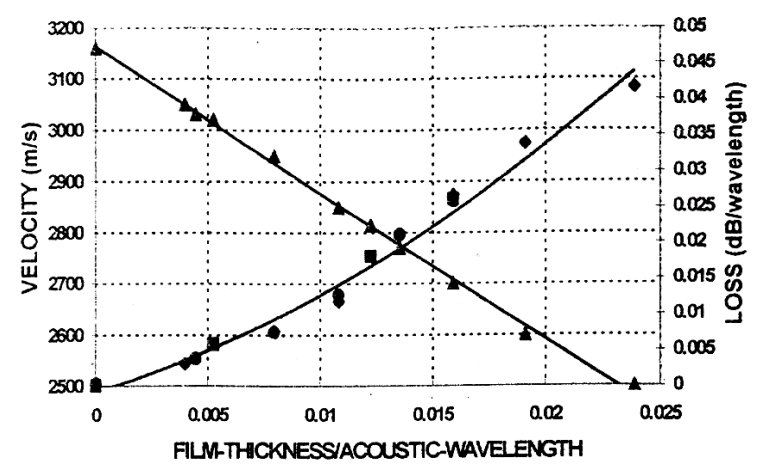

Figure 2. Gold Thin Film on Quartz 
During the initial growth phase $(<5 \mathrm{~nm})$, where discontinuous metal islands were formed, there was a small increase in attenuation and a slight decrease in phase velocity. As the metal islands connect and the electrical resistance decreases, a region of rapid velocity change and increased attenuation occurs whose magnitudes are dependent upon the coupling factor of the piezoelectric substrate. This occurs for film thickness in the range of 5 to $10 \mathrm{~nm}$ and is associated with the acoustoelectric effect. Beyond $10 \mathrm{~nm}$ the velocity and propagation loss come under the influence of the mass and elastic properties of the film. The study was based on depositing gold thin films in the bare area between the transducers. In this paper we investigate the effect of depositing gold thin film on the entire SAW device which includes direct deposition on the transducers.

\section{Coating with SEM Specimen Ion Coater}

To first try if indeed SAWR frequency can be trimmed with simple gold thin film addition, we used a laboratory Teiko IB-3 ion coater to deposit the film (Fig. 3). The ion sputtering coater is routinely used to deposit conductive film on specimens for observing in our in-house Scanning Electron Microscope (SEM). With applying more than a few hundred volt between electrodes faced each other in vacuum of $1 \times 10^{-1} \sim 5 \times 10^{-2}$ Torr, the glow discharge occurs and small amount of gases are ionized. By keeping conductive coating metal electronegative potential, ionized gases are accelerated in electric field and clash with metal surface, then sputtering is generated. Emitted metal atoms clash with gas molecules and then reach a specimen from random direction. Thus a homogeneous thin film is formed.

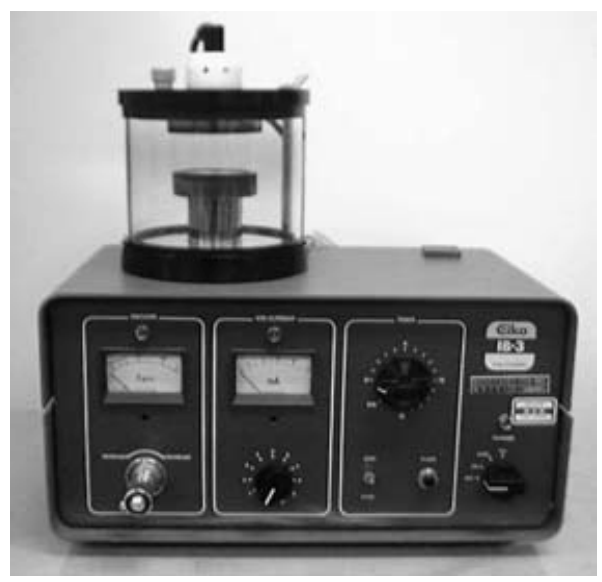

Figure 3. Teiko IB-3 Ion Sputtering Coater

The test vehicle used was a $622 \mathrm{MHz}$ 1-port SAWR using Y-X quartz with typical performance- $\mathrm{Rm}=14 \mathrm{Ohm}, \mathrm{Cm}=1.8 \mathrm{fF}$, Co $=2.2 \mathrm{pF}, \mathrm{IL}=2 \mathrm{~dB}$ and unloaded $\mathrm{Q}=10,000$. The line-width is $\sim 1.3 \mu \mathrm{m}$ and the $\mathrm{Al}$ finger thickness is $\sim 1000 \AA$. The SAWR is set inside a $3.8 \mathrm{~mm} \times 3.8 \mathrm{~mm}$ leadless chip carrier (Fig. 4). No specific control was employed. The SAWR was simply repeatedly measured, inserted into the chamber and coated, removed from the chamber, and measured. Fig. 5 shows the frequency and insertion loss changes in 7 cycles. In the end, frequency trimmed was about $2 \mathrm{MHz}$ (>3000 ppm) and the insertion loss increased by less than $1 \mathrm{~dB}$. The experiment showed no drastic performance deterioration observed after even more than $3000 \mathrm{ppm}$ frequency drift.

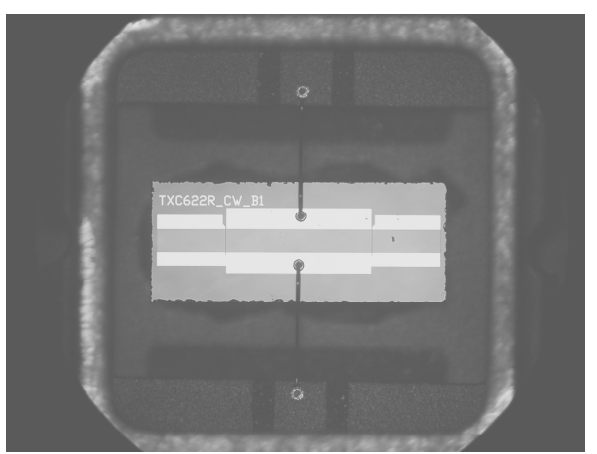

Figure 4. $622 \mathrm{MHz} 1$-port SAWR in $3.8 \mathrm{mmx} 3.8 \mathrm{~mm} \mathrm{LCC}$

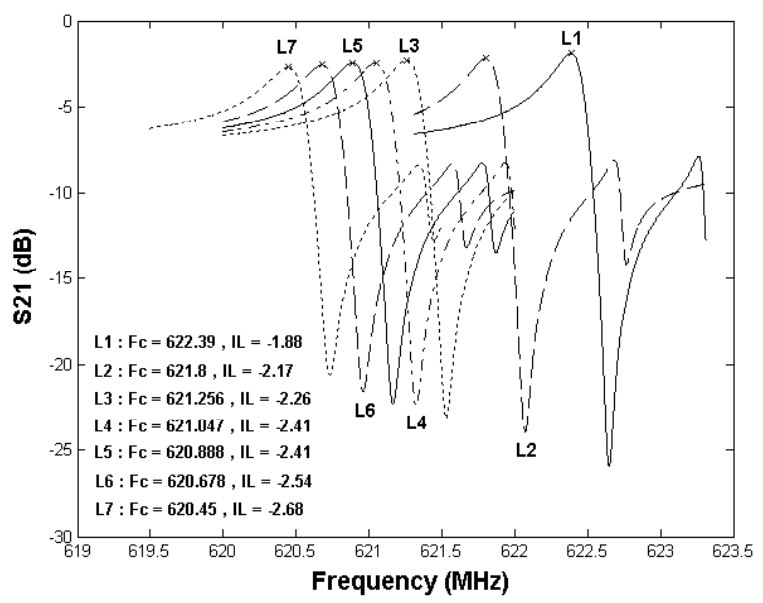

Figure 5. Frequency and Insertion Loss Changes of a $622 \mathrm{MHz}$ SAWR upon Gold Thin Film Addition

\section{Coating with S\&A 5520}

The next step was to perform controlled in-situ SAWR frequency trimming using a conventional crystal resonator frequency trimming system (fine tuner). The equipment we chose was an in-house S\&A 5520 crystal resonator frequency trimming system (Fig. 6).

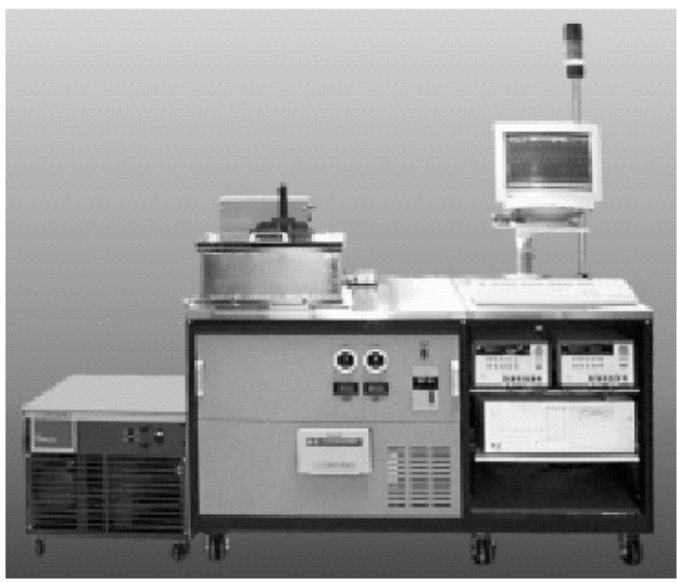

Figure 6. S\&A 5520 Crystal Resonator Frequency Trimming System (Courtesy: Saunders \& Associates, Inc.) 


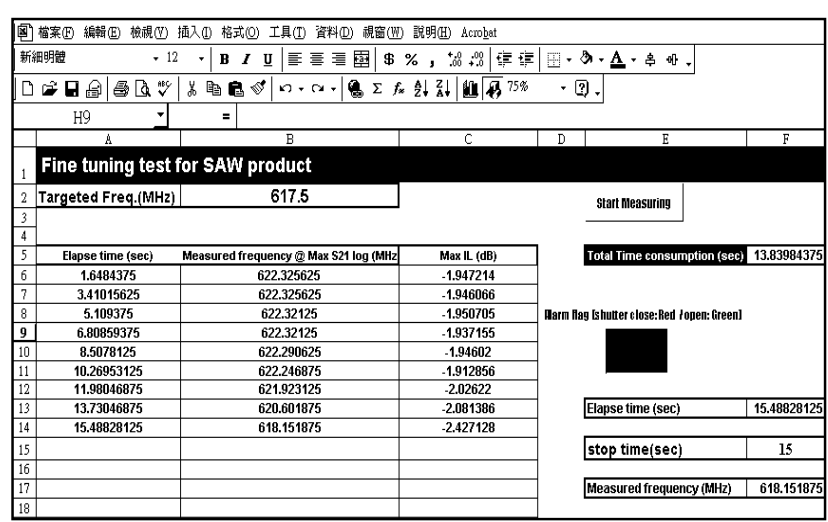

Figure 7. Test Routine to Track In-situ Trimming Process

We slightly modified the system and implemented a test routine so it can measure the $622 \mathrm{MHz}$ SAWR (Fig. 7). The S\&A 5520 employs a simple filament heat evaporation method to flash gold with a maximum power of $3.3 \mathrm{KW}$ at $220 \mathrm{VAC}$ and 15 Amperes. In actual operation, power was recommended to be set between 50 80\% dependent on the filament type used and the facility power applied. Higher filament power settings heat up the depositing material faster but it becomes more difficult to control the trim rate.

We prepared a total of 33 samples and ran them through 3 different percentages of maximum power (Table 1). The modified system configuration rendered the shutter open at the beginning of the process. The shutter was set to close at 15 , 10 , and 8 seconds, respectively.

\begin{tabular}{|c|c|c|}
\hline Samples \# & \% of Max. Power & Duration (sec.) \\
\hline \multirow{2}{*}{6} & \multirow{2}{*}{65} & 15 \\
\cline { 1 - 1 } 6 10 & & 10 \\
\hline $11 \sim 15$ & & 8 \\
\hline $16 \sim 20$ & \multirow{3}{*}{60} & 15 \\
\hline $21 \sim 25$ & & 10 \\
\hline $26 \sim 30$ & & 8 \\
\hline $31 \sim 33$ & 55 & 15 \\
\hline
\end{tabular}

Table 1. 33 Samples at Different \%'s of Power and Shutter Durations

Due to the voluminous amount of data collected, we present here only the frequency and insertion loss changes of the following two cases- $60 \%$ maximum power plus 15 -second shutter duration (Fig. 8) and 65\% maximum power plus 15-second shutter duration (Fig. 9).

Both figures showed that the first few seconds were consumed on filament heating up. No evaporation occurred during that time. At around 9 11 seconds, frequency and insertion loss changes began. At $60 \%$ power, a maximum of $\sim 0.8 \mathrm{MHz}$ was trimmed by the time the shutter closed at 15 seconds. Insertion loss remained steady. At $65 \%$ power, almost $5 \mathrm{MHz}$ was trimmed and the insertion loss increased by $1 \mathrm{~dB}$ when the shutter closed. Clearly, the power setting is critical in controlling the trim rate. In practice, one would need to trim no more than $200 \mathrm{KHz}$ in the case of $622 \mathrm{MHz}$ SAWR $(\sim 300$ ppm) assuming the wafer was fabricated with modest photolithographic process. Additional study is being performed to understand how one can configure the system to control the trim rate effectively.
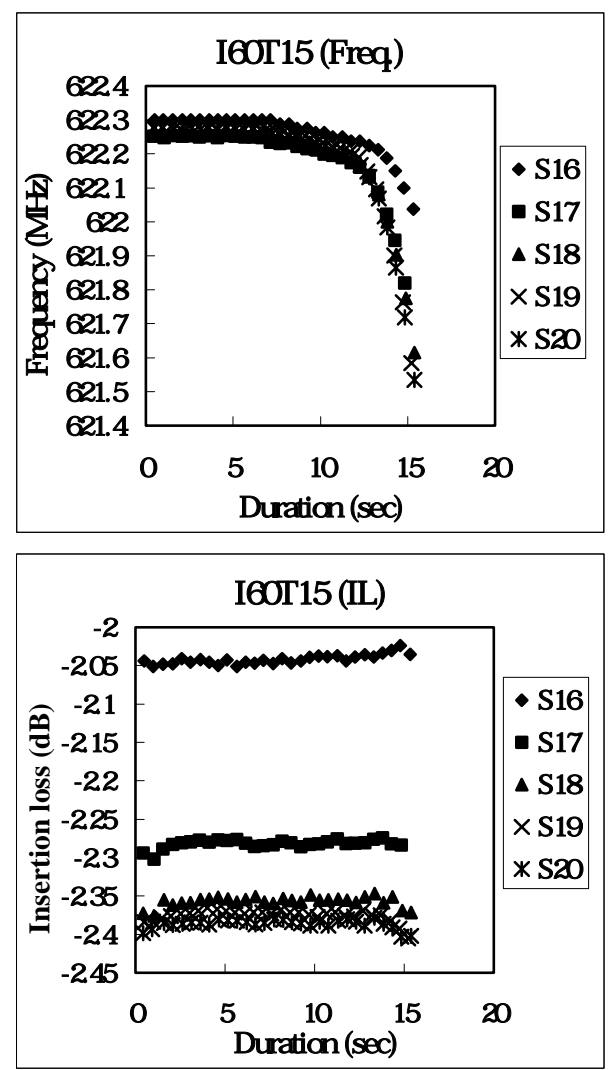

Figure 8. Frequency and Insertion Loss Changes of In-situ Trimming Process- 60\% Power
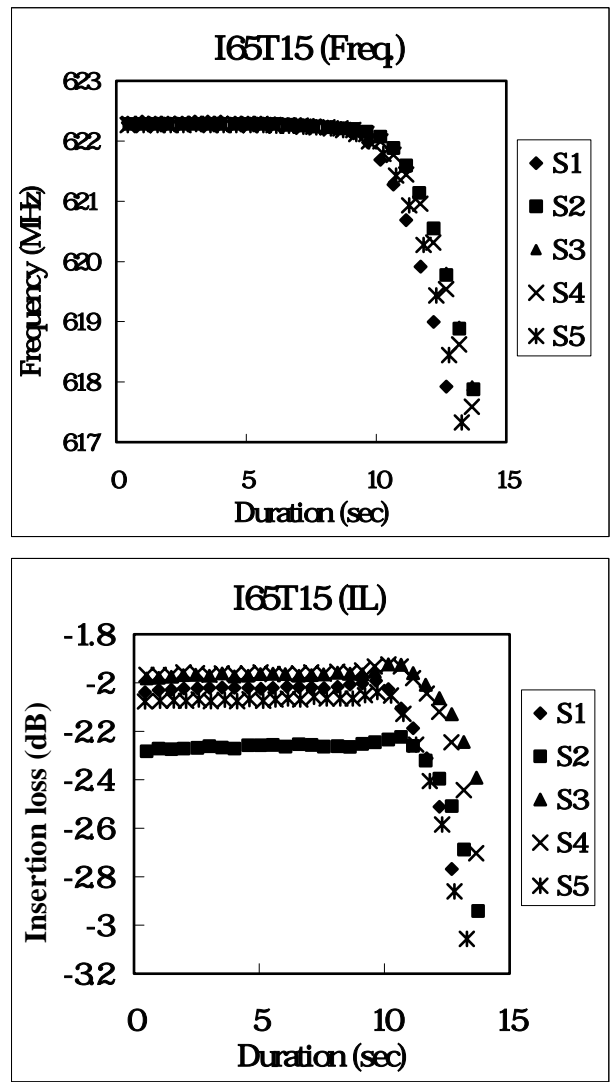

Figure 9. Frequency and Insertion Loss Changes of In-situ Trimming Process- 65\% Power 


\section{SEM Observation and Aging Study}

Using a JEOL JSM-6500-F Scanning Electron Microscope, we observed the finger-quartz region of a SAWR sample prior to and after gold thin film deposition (Figs. 10 and 11). The sample saw a $4.5 \mathrm{MHz}$ drop in frequency and was one of the heavily "coated" samples. It can be seen that gold grains (islands) began to connect in Fig. 11. We have not measured the gold thin film thickness of this sample. No observable gold grains were identified for samples which saw less than 1 $\mathrm{MHz}$ frequency shift.

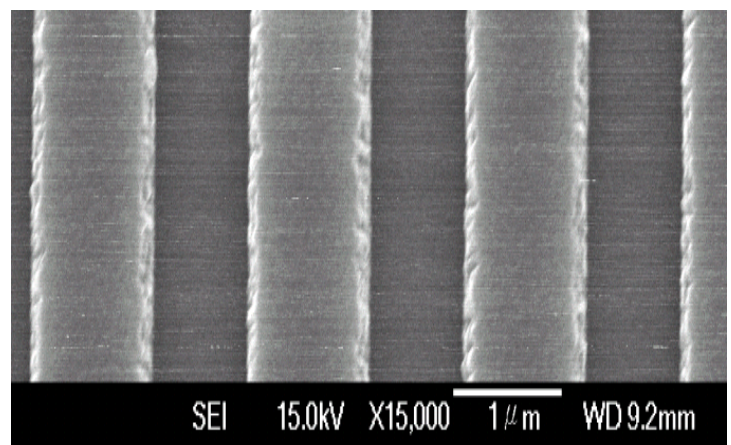

Figure 10. Finger-Quartz Region Prior to Deposition

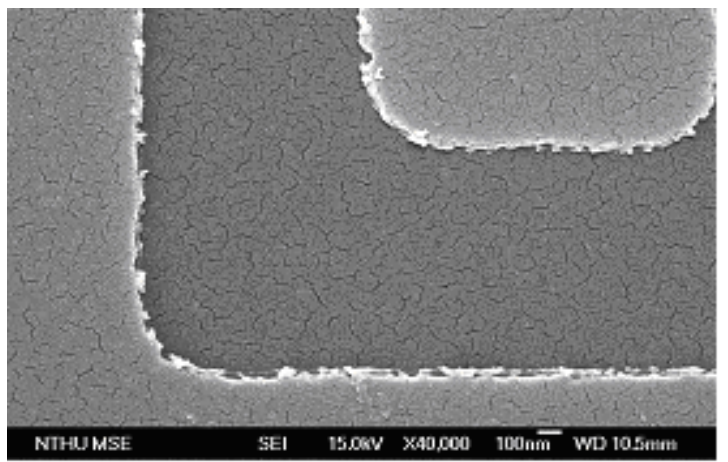

Figure 11. Finger-Quartz Region After Deposition

We subjected the 33 trimmed samples to a quick 48 hours aging at $85^{\circ} \mathrm{C}$. Frequency drift due to aging was plotted against the frequency drift due to deposition (Fig. 12). One can observe that thicker the deposition more aging the device would experience. Thermal shock and extended aging are also being performed to quantify the reliability of this thin film deposition frequency trimming method.

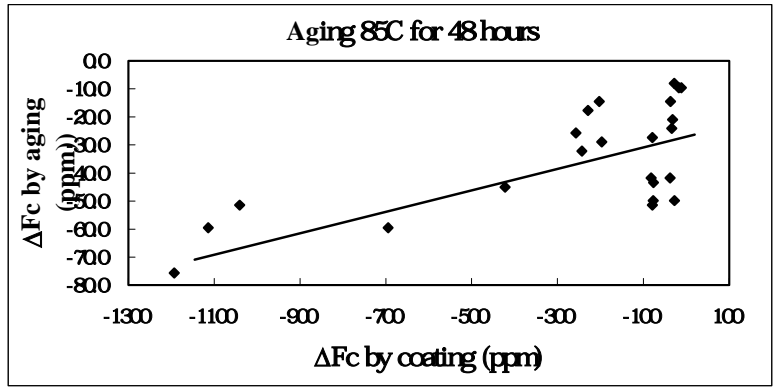

Figure 12. Frequency Drift due to Aging vs Frequency Drift due to Deposition

\section{Discussion}

We demonstrated we could trim assembled SAW resonator (SAWR) by using the conventional crystal resonator fine-tuning process with gold thin film addition. The method provides a wide trimming range and the gold film is thin enough not to short out the SAW transducers so that the SAW resonator's integrity can be maintained. Additional study is ongoing to investigate the trim rate control, mechanical reliability and aging.

\section{Acknowledgement}

We want to thank Mr. Dwane Rose of Saunders \& Associates, Inc. for providing valuable information on the S\&A systems.

\section{References}

1. "Fine Tuning of Narrow-Band SAW Devices Using Dielectric Overlays," C.N. Helmick et al., Proc. 1977 Ultrasonics Symposium, pp. 659-663.

2. "Temperature-Compensated SAW Devices with $\mathrm{SiO}_{2}$ Film Overlays," T.E. Parker and H. Wichansky, J. Appl. Phys. 50 (3), pp. 1360-1369.

3. "High Frequency SAW Filter on Diamond," K. Higaki et al., 1997 MTT-S Digest, pp. 829-832.

4. "Thin-Films for SAW Devices," F.S. Hickernell, Advances in Surface Acoustic Wave Technology, Systems and Applications (Vol. 1), Ed. Clemens C.W. Ruppel and Tor A. Fjeldly, 2000, World Scientific.

5. "Dispersive Rayleigh Waves in Quartz," G. Cambon and C.F. Quate, Electron. Lett., 5 (1969), pp. 402-403.

6. "Attenuation of Dispersive Rayleigh Waves on Quartz," G. Cambon and M. Rouzeyre, Electron. Lett., 6 (1970), pp. 539-540. 